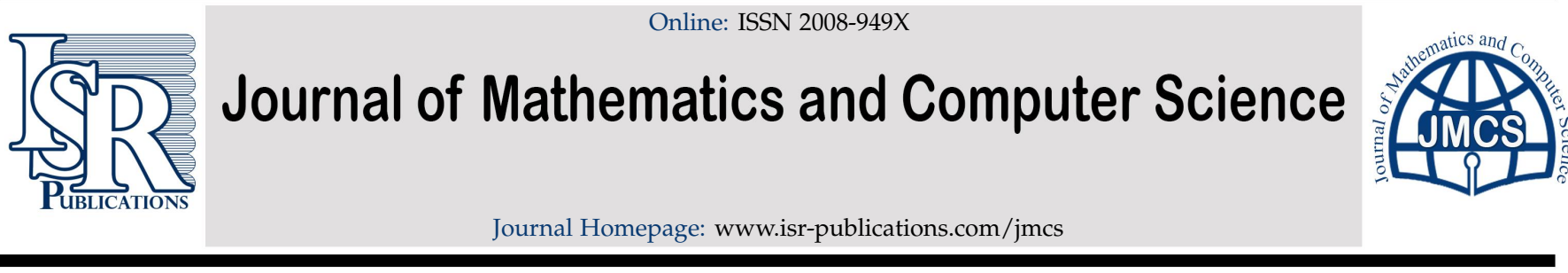

\title{
A fixed point approach to the stability of a general quartic functional equation
}

\author{
Yang-Hi Lee ${ }^{\mathrm{a}}$, Soon-Mo Jung ${ }^{\mathrm{b}, *}$ \\ ${ }^{a}$ Department of Mathematics Education, Gongju National University of Education, Gongju 32553, Republic of Korea. \\ ${ }^{b}$ Mathematics Section, College of Science and Technology, Hongik University, 30016 Sejong, Republic of Korea.
}

\begin{abstract}
In this paper, we study the generalized Hyers-Ulam stability of the quartic functional equation

$$
f(x+3 y)-5 f(x+2 y)+10 f(x+y)-10 f(x)+5 f(x-y)-f(x-2 y)=0,
$$
\end{abstract}

by applying the fixed point method.

Keywords: Fixed point method, fixed point, stability, generalized Hyers-Ulam stability, quartic functional equation. 2010 MSC: 39B82, 39B52, 47H10, 47N99.

(C)2020 All rights reserved.

\section{Introduction}

The stability problem of the functional equations stems from Ulam's well known question about the stability of group homomorphisms (see [16]):

Let $\mathrm{G}_{1}$ be a group and let $\mathrm{G}_{2}$ be a metric group with the metric $\mathrm{d}(\cdot, \cdot)$. Given $\varepsilon>0$, does there exist an $\delta>0$ such that if a function $\mathrm{h}: \mathrm{G}_{1} \rightarrow \mathrm{G}_{2}$ satisfies the inequality $\mathrm{d}(\mathrm{h}(\mathrm{xy}), \mathrm{h}(\mathrm{x}) \mathrm{h}(\mathrm{y}))<\delta$ for all $x, y \in \mathrm{G}_{1}$, then there exists a homomorphism $\mathrm{H}: \mathrm{G}_{1} \rightarrow \mathrm{G}_{2}$ with $\mathrm{d}(\mathrm{h}(\mathrm{x}), \mathrm{H}(\mathrm{x}))<\varepsilon$ for all $\mathrm{x} \in \mathrm{G}_{1}$ ?

In 1941, Hyers [9] affirmatively answered the Ulam's question for the additive functional equation under the assumption that $G_{1}$ and $G_{2}$ are Banach spaces. Indeed, Hyers proved that each solution of inequality $\|f(x+y)-f(x)-f(y)\| \leqslant \varepsilon$, can be approximated by an exact solution (an additive function). In this case, we say that the Cauchy additive functional equation, $f(x+y)=f(x)+f(y)$, satisfies the Hyers-Ulam stability or it is stable in the sense of Hyers and Ulam.

Since then, the stability of various functional equations has been extensively studied by a number of mathematicians (e.g., see $[1-3,8,10-12,15]$ and the references therein).

\footnotetext{
*Corresponding author

Email addresses: yanghi2@hanmail .net (Yang-Hi Lee), smjung@hongik.ac.kr (Soon-Mo Jung)

doi: $10.22436 /$ jmcs.020.03.03
}

Received: 2019-09-20 Revised: 2019-10-11 Accepted: 2019-10-15 
Every solution of the Cauchy additive functional equation

$$
f(x+y)-f(x)-f(y)=0,
$$

is called an additive mapping, and each solution of the quadratic functional equation

$$
f(x+y)+f(x-y)-2 f(x)-2 f(y)=0,
$$

is called a quadratic mapping.

In this paper, we will deal with a special type of quartic functional equation

$$
\operatorname{Df}(x, y)=0,
$$

where we define

$$
D f(x, y):=f(x+3 y)-5 f(x+2 y)+10 f(x+y)-10 f(x)+5 f(x-y)-f(x-2 y),
$$

for all $(x, y)$ in the domain of $f$. It is not difficult to verify that the mapping $f(x)=a x^{4}+b x^{3}+c x^{2}+d x+e$ is a solution to this equation when $a, b, c, d, e$ are real constants. We remind that a mapping $f$ is called a quartic mapping provided that there exist real numbers $a, b, c, d$, e so that $f(x)=a x^{4}+b x^{3}+c x^{2}+d x+e$. For the case of $a=1$ and $b=-1$, Lee [14] proved the stability of (1.1) for restricted domains in Banach spaces.

In this paper, we will prove that every solution of functional equation (1.1) with $f(0)=0$ is a quartic mapping and we will introduce a strictly contractive mapping which allows us to use the fixed point theory in the sense of Cădariu and Radu [4-6]. And then we will adopt the fixed point method for proving the stability of the functional equation (1.1). The key point is that starting from the mapping f satisfying (1.1) approximately, we construct the exact solution F of (1.1) explicitly by using either the formula

$$
F(x)=\lim _{n \rightarrow \infty}\left(\sum_{i=0}^{n}{ }_{n} C_{i} \frac{(-1)^{n-i} 30^{i}}{81^{n}} f_{o}\left(3^{2 n-i} x\right)+\sum_{i=0}^{n}{ }_{n} C_{i} \frac{(-1)^{n-i} 90^{i}}{729^{n}} f_{e}\left(3^{2 n-i} x\right)\right),
$$

or

$$
F(x)=\lim _{n \rightarrow \infty} \sum_{i=0}^{n}{ }_{n} C_{i}\left(30^{i}(-81)^{n-i} f_{o}\left(\frac{x}{3^{2 n-i}}\right)+90^{i}(-729)^{n-i} f_{e}\left(\frac{x}{3^{2 n-i}}\right)\right) .
$$

In the last step we will approximate $f$ with $F$.

\section{Main results}

We will use Margolis and Diaz's theorem in fixed point theory. Recently, this theorem has been widely used to prove the stability of various functional equations.

Theorem 2.1 ([7]). Assume that $(\mathrm{S}, \mathrm{d})$ is a complete generalized metric space which means that the metric $\mathrm{d}$ may assume infinite values. Moreover, assume that $\mathrm{J}: \mathrm{S} \rightarrow \mathrm{S}$ is a strictly contractive mapping with the Lipschitz constant $0<\mathrm{L}<1$. Then, for each given element $\mathrm{x} \in \mathrm{S}$, either

$$
\mathrm{d}\left(\mathrm{J}^{\mathrm{n}} \mathrm{x}, \mathrm{J}^{\mathrm{n}+1} \mathrm{x}\right)=\infty, \quad \forall \mathrm{n} \in \mathbb{N} \cup\{0\},
$$

or there exists a $\mathrm{k} \in \mathbb{N} \cup\{0\}$ such that

(1) $d\left(J^{n} x, J^{n+1} x\right)<\infty$, for all $n \geqslant k$;

(2) the sequence $\left\{\mathrm{J}^{\mathrm{n}} \mathrm{x}\right\}$ is convergent to a fixed point $\mathrm{y}^{*}$ of $\mathrm{J}$;

(3) $\mathrm{y}^{*}$ is the unique fixed point of $\mathrm{J}$ in $\mathrm{T}:=\left\{\mathrm{y} \in \mathrm{S} \mid \mathrm{d}\left(\mathrm{J}^{\mathrm{k}} \mathrm{x}, \mathrm{y}\right)<\infty\right\}$; 
(4) $d\left(y, y^{*}\right) \leqslant \frac{1}{1-L} d(y, J y)$, for all $y \in T$.

Throughout this paper, let $V$ and $W$ be real vector spaces and let $Y$ be a real Banach space. For a given mapping $f: V \rightarrow W$, we use the following abbreviations

$$
f_{e}(x):=\frac{1}{2}(f(x)+f(-x)) \text { and } f_{o}(x):=\frac{1}{2}(f(x)-f(-x)),
$$

for all $x \in V$.

In the following theorem, we prove the generalized Hyers-Ulam stability of the functional equation (1.1) by using the fixed point method (Theorem 2.1).

Theorem 2.2. Assume that a mapping $\varphi: \mathrm{V} \times \mathrm{V} \rightarrow[0, \infty)$ satisfies the condition

$$
\varphi(3 x, 3 y) \leqslant(\sqrt{306}-15) \operatorname{L} \varphi(x, y),
$$

for all $\mathrm{x}, \mathrm{y} \in \mathrm{V}$ and for some constant $0<\mathrm{L}<1$ and let

$$
\Phi(x)=\frac{1}{729}\left(10 \varphi_{e}(0,3 x)+42 \varphi_{e}(0,2 x)+480 \varphi_{e}(0, x)+180 \varphi_{e}(x, x)\right),
$$

where

$$
\varphi_{e}(x, y)=\frac{1}{2}(\varphi(x, y)+\varphi(-x,-y)) .
$$

If a mapping $\mathrm{f}: \mathrm{V} \rightarrow \mathrm{Y}$ with $\mathrm{f}(0)=0$ satisfies the inequality

$$
\|\operatorname{Df}(x, y)\| \leqslant \varphi(x, y),
$$

for all $x, y \in V$, then there exists a unique solution $\mathrm{F}: \mathrm{V} \rightarrow \mathrm{Y}$ of (1.1) such that

$$
\|f(x)-F(x)\| \leqslant \frac{1}{1-L} \Phi(x),
$$

for all $\mathrm{x} \in \mathrm{V}$. In particular, $\mathrm{F}$ is represented by

$$
F(x)=\lim _{n \rightarrow \infty} \sum_{i=0}^{n}{ }_{n} C_{i}\left(\frac{(-1)^{n-i} 30^{i}}{81^{n}} f_{o}\left(3^{2 n-i} x\right)+\frac{(-1)^{n-i} 90^{i}}{729^{n}} f_{e}\left(3^{2 n-i} x\right)\right),
$$

for all $\mathrm{x} \in \mathrm{V}$.

Proof. Let $\mathrm{S}$ be the set of all functions $\mathrm{g}: \mathrm{V} \rightarrow \mathrm{Y}$ with $\mathrm{g}(0)=0$. We introduce the generalized metric $\mathrm{d}$ in $S$ defined by

$$
d(g, h)=\inf \{K \geqslant 0 \mid\|g(x)-h(x)\| \leqslant K \Phi(x), \text { for all } x \in V\} .
$$

It is easy to verify that $(S, d)$ is a complete generalized metric space (see [6, Theorem 2.5] or the proof of [13, Theorem 3.1]).

Now we consider the mapping $J: S \rightarrow S$, which is defined by

$$
J g(x)=\frac{180}{729} g(3 x)-\frac{90}{729} g(-3 x)-\frac{5}{729} g(9 x)+\frac{4}{729} g(-9 x),
$$

for all $x \in V$. Applying mathematical induction, we can prove the equality

$$
J^{n} g(x)=\sum_{i=0}^{n}{ }_{n} C_{i}\left(\frac{(-1)^{n-i} 30^{i}}{81^{n}} g_{o}\left(3^{2 n-i} x\right)+\frac{(-1)^{n-i} 90^{i}}{729^{n}} g_{e}\left(3^{2 n-i} x\right)\right),
$$

for all $\mathrm{n} \in \mathbb{N} \cup\{0\}$ and $x \in \mathrm{V}$. 
Indeed it follows from (2.6) that

$$
\mathrm{J}^{0} \mathrm{~g}(\mathrm{x})=\mathrm{g}(\mathrm{x})=\mathrm{g}_{\mathrm{o}}(\mathrm{x})+\mathrm{g}_{\mathrm{e}}(x),
$$

and using the oddness and the evenness of $g_{o}$ and $g_{e}$ of (2.6) we get

$$
\begin{aligned}
J^{n+1} g(x)= & J\left(J^{n} g(x)\right) \\
= & \frac{135}{729} J^{n} g(3 x)-\frac{135}{729} J^{n} g(-3 x)+\frac{45}{729} J^{n} g(3 x)+\frac{45}{729} J^{n} g(-3 x) \\
& -\frac{9}{1458} J^{n} g(9 x)+\frac{9}{1458} J^{n} g(-9 x)-\frac{1}{1458} J^{n} g(9 x)-\frac{1}{1458} J^{n} g(-9 x) \\
= & \frac{270}{729} \sum_{i=0}^{n}{ }_{n} C_{i} \frac{(-1)^{n-i} 30^{i}}{81^{n}} g_{o}\left(3^{2 n+1-i} x\right) \\
& +\frac{90}{729} \sum_{i=0}^{n}{ }_{n} C_{i} \frac{(-1)^{n-i} 90^{i}}{729^{n}} g_{e}\left(3^{2 n+1-i} x\right) \\
& -\frac{9}{729} \sum_{i=0}^{n}{ }_{n} C_{i} \frac{(-1)^{n-i} 30^{i}}{81^{n}} g_{o}\left(3^{2 n+2-i} x\right) \\
& -\frac{1}{729} \sum_{i=0}^{n}{ }_{n} C_{i} \frac{(-1)^{n-i} 90^{i}}{729^{n}} g_{e}\left(3^{2 n+2-i} x\right) \\
= & \frac{270}{729} \sum_{i=1}^{n+1}{ }_{n} C_{i-1} \frac{(-1)^{n+1-i} 30^{i-1}}{81^{n}} g_{o}\left(3^{2 n+2-i} x\right) \\
& +\frac{90}{729} \sum_{i=1}^{n+1}{ }_{n} C_{i-1} \frac{(-1)^{n+1-i} 90^{i-1}}{729^{n}} g_{e}\left(3^{2 n+2-i} x\right) \\
& -\frac{9}{729} \sum_{i=0}^{n}{ }_{n} C_{i} \frac{(-1)^{n-i} 30^{i}}{81^{n}} g_{o}\left(3^{2 n+2-i} x\right) \\
& -\frac{1}{729} \sum_{i=0}^{n}{ }_{n} C_{i} \frac{(-1)^{n-i} 90^{i}}{729^{n}} g_{e}\left(3^{2 n+2-i} x\right) .
\end{aligned}
$$

Furthermore, by using the well known formula ${ }_{n} C_{i-1}+{ }_{n} C_{i}={ }_{n+1} C_{i}$, we obtain

$$
\begin{aligned}
J^{n+1} g(x)= & \sum_{i=1}^{n+1}{ }_{n} C_{i-1} \frac{(-1)^{n+1-i} 30^{i}}{81^{n+1}} g_{o}\left(3^{2 n+2-i} x\right) \\
& +\sum_{i=1}^{n+1}{ }_{n} C_{i-1} \frac{(-1)^{n+1-i} 90^{i}}{729^{n+1}} g_{e}\left(3^{2 n+2-i} x\right) \\
& -\sum_{i=0}^{n}{ }_{n} C_{i} \frac{(-1)^{n-i} 30^{i}}{81^{n+1}} g_{o}\left(3^{2 n+2-i} x\right) \\
& -\sum_{i=0}^{n}{ }_{n} C_{i} \frac{(-1)^{n-i} 90^{i}}{729^{n+1}} g_{e}\left(3^{2 n+2-i} x\right) \\
= & \sum_{i=0}^{n+1}{ }^{n+1} C_{i}\left(\frac{(-1)^{n+1-i} 30^{i}}{81^{n+1}} g_{o}\left(3^{2 n+2-i} x\right)+\frac{(-1)^{n+1-i} 90^{i}}{729^{n+1}} g_{e}\left(3^{2 n+2-i} x\right)\right)
\end{aligned}
$$

which implies the validity of (2.6) for all $n \in \mathbb{N} \cup\{0\}$. 
Assume $g, h \in S$ and suppose $K \in[0, \infty]$ is an arbitrary constant satisfying $d(g, h) \leqslant K$. From the definitions of $\mathrm{d}$ and $\Phi$ and by (2.1), we note that $\Phi(-x)=\Phi(x)$ and $\Phi(3 x) \leqslant(\sqrt{306}-15) \operatorname{L} \Phi(x)$ for all $x \in \mathrm{V}$. Hence, we further get

$$
\begin{aligned}
\|J g(x)-J h(x)\| \leqslant & \frac{5}{729}\|g(9 x)-h(9 x)\|+\frac{4}{729}\|g(-9 x)-h(-9 x)\| \\
& +\frac{180}{729}\|g(3 x)-h(3 x)\|+\frac{90}{729}\|g(-3 x)-h(-3 x)\| \\
\leqslant & K\left(\frac{9}{729} \Phi(9 x)+\frac{270}{729} \Phi(3 x)\right) \\
\leqslant & K\left(\frac{\sqrt{306}-15}{81} \mathrm{~L} \Phi(3 x)+\frac{30}{81} \Phi(3 x)\right) \\
\leqslant & K \frac{(\sqrt{306}-15)^{2}+30(\sqrt{306}-15)}{81} \mathrm{~L} \Phi(x) \\
\leqslant & \operatorname{LK} \Phi(x),
\end{aligned}
$$

for all $x \in V$, which implies that

$$
d(J g, J h) \leqslant \operatorname{Ld}(g, h)
$$

for any $g, h \in S$. That is, $J$ is a strictly contractive self-mapping of $S$ with the Lipschitz constant $L$.

Moreover, it follows from (1.1) that

$$
\begin{aligned}
D f_{e}(x, y)= & \frac{1}{2} f(x+3 y)+\frac{1}{2} f(-x-3 y)-\frac{5}{2} f(x+2 y)-\frac{5}{2} f(-x-2 y) \\
& +5 f(x+y)+5 f(-x-y)-5 f(x)-5 f(-x) \\
& +\frac{5}{2} f(x-y)+\frac{5}{2} f(-x+y)-\frac{1}{2} f(x-2 y)-\frac{1}{2} f(-x+2 y)
\end{aligned}
$$

and

$$
\begin{aligned}
D f_{o}(x, y)= & \frac{1}{2} f(x+3 y)-\frac{1}{2} f(-x-3 y)-\frac{5}{2} f(x+2 y)+\frac{5}{2} f(-x-2 y) \\
& +5 f(x+y)-5 f(-x-y)-5 f(x)+5 f(-x) \\
& +\frac{5}{2} f(x-y)-\frac{5}{2} f(-x+y)-\frac{1}{2} f(x-2 y)+\frac{1}{2} f(-x+2 y)
\end{aligned}
$$

for all $x, y \in V$. On account of (2.5), and by a long and tedious calculation, we obtain

$$
\begin{aligned}
& \frac{1}{729}\left(D f_{e}(0,3 x)+6 D f_{e}(0,2 x)+75 D f_{e}(0, x)+36 D f_{e}(x, x)\right) \\
& +\frac{1}{81}\left(D f_{o}(0,3 x)+4 D f_{o}(0,2 x)+45 D f_{o}(0, x)+16 D f_{o}(x, x)\right) \\
& \quad=f(x)-J f(x)
\end{aligned}
$$

Hence, by (2.2) and since $\left\|D f_{o}(x, y)\right\| \leqslant \varphi_{e}(x, y)$ and $\left\|D f_{e}(x, y)\right\| \leqslant \varphi_{e}(x, y)$, we see that

$$
\begin{aligned}
\|f(x)-J f(x)\|= & \frac{1}{729}\left\|D f_{e}(0,3 x)+6 D f_{e}(0,2 x)+75 D f_{e}(0, x)+36 D f_{e}(x, x)\right\| \\
& +\frac{1}{81}\left\|D f_{o}(0,3 x)+4 D f_{o}(0,2 x)+45 D f_{o}(0, x)+16 D f_{o}(x, x)\right\| \\
\leqslant & \frac{10 \varphi_{e}(0,3 x)+42 \varphi_{e}(0,2 x)+480 \varphi_{e}(0, x)+180 \varphi_{e}(x, x)}{729} \\
= & \Phi(x)
\end{aligned}
$$


for all $x \in V$. It implies that $d(f, J f) \leqslant 1<\infty$ from the definition of $d$.

Therefore, according to Theorem 2.1, the sequence $\left\{\mathrm{J}^{\mathrm{n}} \mathrm{f}\right\}$ converges to the unique fixed point $\mathrm{F}: \mathrm{V} \rightarrow \mathrm{Y}$ of $J$ in the set $T=\{g \in S \mid d(f, g)<\infty\}$, which is represented by (2.4) for all $x \in V$. Further, it follows from Theorem 2.1 (4) that

$$
d(f, F) \leqslant \frac{1}{1-L} d(f, J f) \leqslant \frac{1}{1-L^{\prime}}
$$

and this inequality implies the validity of (2.3). By the definition of $F$, together with (2.1), (2.2), and (2.6), we have

$$
\begin{aligned}
&\|D F(x, y)\|= \lim _{n \rightarrow \infty}\left\|D J^{n} f(x, y)\right\| \\
&= \lim _{n \rightarrow \infty} \| \sum_{i=0}^{n}{ }_{n} C_{i} \frac{(-1)^{n-i} 30^{i}}{81^{n}} D_{0}\left(3^{2 n-i} x, 3^{2 n-i} y\right) \\
&+\sum_{i=0}^{n}{ }_{n} C_{i} \frac{(-1)^{n-i} 90^{i}}{729^{n}} D f_{e}\left(3^{2 n-i} x, 3^{2 n-i} y\right) \| \\
& \leqslant \lim _{n \rightarrow \infty} \sum_{i=0}^{n}{ }_{n} C_{i}\left(\frac{30^{i}}{81^{n}}+\frac{90^{i}}{729^{n}}\right) \varphi_{e}\left(3^{2 n-i} x, 3^{2 n-i} y\right) \\
& \leqslant \lim _{n \rightarrow \infty} \sum_{i=0}^{n}{ }_{n} C_{i} \frac{30^{i}}{81^{n}} \varphi_{e}\left(3^{2 n-i} x, 3^{2 n-i} y\right) \\
& \leqslant \lim _{n \rightarrow \infty} \sum_{i=0}^{n}{ }_{n} C_{i} \frac{30^{i}(\sqrt{306}-15)^{n-i} L^{n-i}}{81^{n}} \varphi_{e}\left(3^{n} x, 3^{n} y\right) \\
&= 2 \lim _{n \rightarrow \infty} \frac{(30+(\sqrt{306}-15) L)^{n}}{81^{n}} \varphi_{e}\left(3^{n} x, 3^{n} y\right) \\
& \leqslant 2 \lim _{n \rightarrow \infty} \frac{(30+(\sqrt{306}-15))^{n}}{81^{n}} \varphi_{e}\left(3^{n} x, 3^{n} y\right) \\
& \leqslant 2 \lim _{n \rightarrow \infty} \frac{(\sqrt{306}+15)^{n}(\sqrt{306}-15)^{n} L^{n}}{81^{n}} \varphi_{e}(x, y) \\
& \leqslant \lim _{n \rightarrow \infty} 2 L^{n} \varphi_{e}(x, y) \\
&=0,{ }_{i}
\end{aligned}
$$

for all $x, y \in V$, i.e., $F$ is a solution of the functional equation (1.1).

Finally, in view of (2.9) and (2.10), if $F$ is a solution of the functional equation (1.1), then the equality

$$
\begin{aligned}
F(x)-J F(x)= & \frac{1}{729}\left(\operatorname{DF}_{e}(0,3 x)+6 \operatorname{DF}_{e}(0,2 x)+75 \operatorname{DF}_{e}(0, x)+36 \operatorname{DF}_{e}(x, x)\right) \\
& +\frac{1}{81}\left(\operatorname{DF}_{o}(0,3 x)+4 \operatorname{DF}_{o}(0,2 x)+45 \operatorname{DF}_{o}(0, x)+16 \operatorname{DF}_{o}(x, x)\right) \\
= & 0,
\end{aligned}
$$

implies that $\mathrm{F}$ is a fixed point of $\mathrm{J}$.

Roughly speaking, the previous theorem dealt with the generalized Hyers-Ulam stability of the quartic functional equation (1.1) for the case of $\varphi(3 x, 3 y)<3 \varphi(x, y)$.

In the following theorem, we now deal with one of cases for $\varphi(3 x, 3 y)>3 \varphi(x, y)$.

Theorem 2.3. For a given mapping $\mathrm{f}: \mathrm{V} \rightarrow \mathrm{Y}$ with $\mathrm{f}(0)=0$, suppose there exists a mapping $\varphi: \mathrm{V}^{2} \rightarrow[0, \infty)$ such that inequality (2.2) holds for all $x, y \in V$. If there exists a constant $0<\mathrm{L}<1$ such that

$$
\mathrm{L} \varphi(3 x, 3 y) \geqslant \frac{10}{\sqrt{261}-16} \varphi(x, y),
$$


for all $x, y \in V$, then there exists a unique solution $\mathrm{F}: \mathrm{V} \rightarrow \mathrm{Y}$ of (1.1), for which the inequality

$$
\|f(x)-F(x)\| \leqslant \frac{1}{1-L} \Psi(x),
$$

holds for all $\mathrm{x} \in \mathrm{V}$, where

$$
\Psi(x)=2 \varphi_{e}\left(0, \frac{x}{3}\right)+10 \varphi_{e}\left(0, \frac{2 x}{9}\right)+120 \varphi_{e}\left(0, \frac{x}{9}\right)+52 \varphi_{e}\left(\frac{x}{9}, \frac{x}{9}\right) .
$$

In particular, $\mathrm{F}$ is represented by

$$
F(x)=\lim _{n \rightarrow \infty} \sum_{i=0}^{n}{ }_{n} C_{i}\left(30^{n-i}(-81)^{i} f_{o}\left(\frac{x}{3^{2 n-i}}\right)+90^{n-i}(-729)^{i} f_{e}\left(\frac{x}{3^{2 n-i}}\right)\right),
$$

for all $\mathrm{x} \in \mathrm{V}$.

Proof. Let $S$ be the set given in the proof of Theorem 2.2. Similarly as in the proof of Theorem 2.2, we define a generalized metric $d$ in $S$ by

$$
d(g, h)=\inf \{K \geqslant 0 \mid\|g(x)-h(x)\| \leqslant K \Psi(x), \text { for all } x \in V\} .
$$

It is not difficult to verify that $(S, d)$ is a complete generalized metric space (or see the proof of [13, Theorem 3.1]).

Now we consider the mapping J $: S \rightarrow S$ defined by

$$
J g(x)=60 g\left(\frac{x}{3}\right)+30 g\left(\frac{-x}{3}\right)-405 g\left(\frac{x}{9}\right)-324 g\left(\frac{-x}{9}\right),
$$

for all $x \in V$.

As we did in (2.6), applying mathematical induction, we can prove the following equality

$$
J^{n} g(x)=\sum_{i=0}^{n}{ }_{n} C_{i}\left(30^{i}(-81)^{n-i} g_{o}\left(\frac{x}{3^{2 n-i}}\right)+90^{i}(-729)^{n-i} g_{e}\left(\frac{x}{3^{2 n-i}}\right)\right),
$$

for all $n \in \mathbb{N} \cup\{0\}$ and $x \in V$.

Assume $g, h \in S$ and suppose $K \in[0, \infty]$ is an arbitrary constant satisfying $d(g, h) \leqslant K$. From the definition of $d$, we have

$$
\begin{aligned}
\|J g(x)-\operatorname{Jh}(x)\| \leqslant & 60\left\|g\left(\frac{x}{3}\right)-h\left(\frac{x}{3}\right)\right\|+30\left\|g\left(\frac{-x}{3}\right)-h\left(\frac{-x}{3}\right)\right\| \\
& +405\left\|g\left(\frac{x}{9}\right)-h\left(\frac{x}{9}\right)\right\|+324\left\|g\left(\frac{-x}{9}\right)-h\left(\frac{-x}{9}\right)\right\| \\
\leqslant & 729 K \Psi\left(\frac{x}{9}\right)+90 K \Psi\left(\frac{x}{3}\right) \\
\leqslant & \mathrm{L}^{2} \frac{(\sqrt{2753}-45)^{2}}{729} \mathrm{~K} \Psi(x)+90 \frac{\sqrt{2753}-45}{729} \operatorname{LK} \Psi(x) \\
\leqslant & \operatorname{LK} \Psi(x),
\end{aligned}
$$

for all $x \in V$, which implies that

$$
d(J g, J h) \leqslant \operatorname{Ld}(g, h),
$$

for any $g, h \in S$. That is, $J$ is a strictly contractive self-mapping of $S$ with the Lipschitz constant $L$. 
Moreover, as we did in the proof of Theorem 2.2, it follows from (1.1), (2.2), (2.7), and (2.8) that

$$
\begin{aligned}
\|f(x)-J f(x)\| & =\| \operatorname{Df}_{e}\left(0, \frac{x}{3}\right)+6 \operatorname{Df}_{e}\left(0, \frac{2 x}{9}\right)+75 \operatorname{Df}_{e}\left(0, \frac{x}{9}\right)+36 \operatorname{Df}_{e}\left(\frac{x}{9}, \frac{x}{9}\right) \\
& +\operatorname{Df}_{o}\left(0, \frac{x}{3}\right)+4 D f_{o}\left(0, \frac{2 x}{9}\right)+45 \operatorname{Df}_{o}\left(0, \frac{x}{9}\right)+16 \operatorname{Df}_{o}\left(\frac{x}{9}, \frac{x}{9}\right) \| \\
\leqslant & 2 \varphi_{e}\left(0, \frac{x}{3}\right)+10 \varphi_{e}\left(0, \frac{2 x}{9}\right)+120 \varphi_{e}\left(0, \frac{x}{9}\right)+52 \varphi_{e}\left(\frac{x}{9}, \frac{x}{9}\right) \\
= & \Psi(x),
\end{aligned}
$$

for all $x \in V$. It means that $d(f, J f) \leqslant 1<\infty$ from the definition of $d$.

Therefore, according to Theorem 2.1, the sequence $\left\{\mathrm{J}^{\mathrm{n}} \mathrm{f}\right\}$ converges to the unique fixed point $\mathrm{F}: \mathrm{V} \rightarrow \mathrm{Y}$ of $J$ in the set $T=\{g \in S \mid d(f, g)<\infty\}$, which is represented by (2.13) for all $x \in V$. Notice that

$$
d(f, F) \leqslant \frac{1}{1-L} d(f, J f) \leqslant \frac{1}{1-L^{\prime}}
$$

and this inequality implies (2.12). By the definition of $F$, together with (2.2) and (2.11), we get

$$
\begin{aligned}
\|D F(x, y)\| & =\lim _{n \rightarrow \infty}\left\|D J^{n} f(x, y)\right\| \\
= & \lim _{n \rightarrow \infty} \| \sum_{i=0}^{n}{ }_{n} C_{i} 30^{i}(-81)^{n-i} f_{o}\left(\frac{x}{3^{2 n-i}}, \frac{y}{3^{2 n-i}}\right) \\
& \quad+\sum_{i=0}^{n}{ }_{n} C_{i} 90^{i}(-729)^{n-i} f_{e}\left(\frac{x}{3^{2 n-i}}, \frac{y}{3^{2 n-i}}\right) \| \\
\leqslant & \lim _{n \rightarrow \infty} \sum_{i=0}^{n}{ }_{n} C_{i}\left(30^{i} 81^{n-i}+90^{n-i} 729^{i}\right) \varphi_{e}\left(\frac{x}{3^{2 n-i}}, \frac{y}{3^{2 n-i}}\right) \\
\leqslant & 2 \lim _{n \rightarrow \infty} \sum_{i=0}^{n}{ }_{n} C_{i} 90^{i} 729^{n-i} \varphi_{e}\left(\frac{x}{3^{2 n-i}}, \frac{y}{3^{2 n-i}}\right) \\
\leqslant & 2 \lim _{n \rightarrow \infty} \sum_{i=0}^{n}{ }_{n} C_{i} 90^{i}(\sqrt{2753}-45)^{n-i} L^{n-i} \varphi_{e}\left(\frac{x}{3^{n}}, \frac{y}{3^{n}}\right) \\
\leqslant & 2 \lim _{n \rightarrow \infty}(90+(\sqrt{2753}-45) L)^{n} \varphi_{e}\left(\frac{x}{3^{n}}, \frac{y}{3^{n}}\right) \\
\leqslant & 2 \lim _{n \rightarrow \infty} \frac{(\sqrt{2753}+45)^{n}(\sqrt{2753}-45)^{n}}{729^{n}} L^{n} \varphi_{e}(x, y) \\
= & 2 \lim _{n \rightarrow \infty} L^{n} \varphi_{e}(x, y) \\
= & 0,
\end{aligned}
$$

for all $x, y \in V$, i.e., $F$ is a solution of functional equation (1.1).

Finally, we notice that if $F$ is a solution of functional equation (1.1), then it follows from the equality

$$
\begin{aligned}
\mathrm{F}(x)-\mathrm{JF}(x)= & \mathrm{DF}_{e}\left(0, \frac{x}{3}\right)+6 \mathrm{DF}_{e}\left(0, \frac{2 x}{9}\right)+75 \mathrm{DF}_{e}\left(0, \frac{x}{9}\right)+36 \mathrm{DF}_{e}\left(\frac{x}{9}, \frac{x}{9}\right) \\
& +\mathrm{DF}_{\mathrm{o}}\left(0, \frac{x}{3}\right)+4 \mathrm{DF}_{\mathrm{o}}\left(0, \frac{2 x}{9}\right)+45 \mathrm{DF}_{\mathrm{o}}\left(0, \frac{x}{9}\right)+16 \mathrm{DF}_{\mathrm{o}}\left(\frac{x}{9}, \frac{x}{9}\right),
\end{aligned}
$$

that $\mathrm{F}$ is a fixed point of $\mathrm{J}$. 


\section{Acknowledgment}

Soon-Mo Jung was supported by Basic Science Research Program through the National Research Foundation of Korea (NRF) funded by the Ministry of Education (No. 2016R1D1A1B03931061).

\section{References}

[1] M. Almahalebi, On the stability of a generalization of Jensen functional equation, Acta Math. Hungar., 154 (2018), 187-198. 1

[2] S. Alshybania, S. M. Vaezpoura, R. Saadati, Generalized Hyers-Ulam stability of sextic functional equation in random normed spaces, J. Comput. Anal. Appl., 24 (2018), 370-381.

[3] J. Brzḑek, E. Karapinar, A. Petruşel, A fixed point theorem and the Ulam stability in generalized dq-metric spaces, J. Math. Anal. Appl., 467 (2018), 501-520. 1

[4] L. Cădariu, V. Radu, Fixed points and the stability of Jensen's functional equation, JIPAM. J. Inequal. Pure Appl. Math., 4 (2003), 7 pages. 1

[5] L. Cădariu, V. Radu, Fixed points and the stability of quadratic functional equations, An. Univ. Timisoara Ser. Mat.Inform., 41 (2003), 25-48.

[6] L. Cădariu, V. Radu, On the stability of the Cauchy functional equation: a fixed point approach in Iteration Theory, Grazer Math. Ber., 346 (2004), 43-52. 1, 2

[7] J. B. Diaz, B. Margolis, A fixed point theorem of the alternative for contractions on a generalized complete metric space, Bull. Amer. Math. Soc., 74 (1968), 305-309. 2.1

[8] P. Găvruta, A generalization of the Hyers-Ulam-Rassias stability of approximately additive mappings, J. Math. Anal. Appl., 184 (1994), 431-436. 1

[9] D. H. Hyers, On the stability of the linear functional equation, Proc. Nat. Acad. Sci. U.S.A., 27 (1941), 222-224. 1

[10] D. H. Hyers, G. Isac, T. M. Rassias, Stability of Functional Equations in Several Variables, Birkäuser, Boston, (1998). 1

[11] D. H. Hyers, T. M. Rassias, Approximate homomorphisms, Aequationes Math., 44 (1992), 125-153.

[12] S.-M. Jung, Hyers-Ulam-Rassias stability of functional equations in nonlinear analysis, Springer, New York, (2011). 1

[13] S.-M. Jung, T.-S. Kim, A fixed point approach to the stability of the cubic functional equation, Bol. Soc. Mat. Mexicana (3), 12 (2006), 51-57. 2, 2

[14] Y.-H. Lee, Hyers-Ulam-Rassias stability of a quadratic-additive type functional equation on a restricted domain, Int. J. Math. Anal. (Ruse), 7 (2013), 2745-2752. 1

[15] T. M. Rassias, On the stability of linear mappings in Banach spaces, Proc. Amer. Math. Soc., 72 (1978), 297-300. 1

[16] S. M. Ulam, A Collection of Mathematical Problems, Interscience Publ., New York-London, (1960). 1 